\title{
A WAVE-MECHANICAL TREATMENT OF THE MILLS-NIXON EFFECT.
}

\author{
By L. E. Sutton and L. Pauling.
}

Received 8th April, 1935.

\section{Contribution No. 444 from the Gates Chemical Laboratory, California Institute of Technology, Pasadena, California.}

In 1930, W. H. Mills and I. G. Nixon ${ }^{1}$ showed that by attaching saturated carbon rings of different sizes to two ortho valencies of a benzene molecule it is possible to make the benzene ring react as though it had either one or other of the two Kekulé structures. Thus, they found that 5 -hydroxyhydrindene undergoes substitution by bromine or by diazo residues in the 6 position, and so behaves as though the benzene ring in this compound had that Kekule structure with the single bond common to the two rings (formula I), whereas ar-tetrahydro- $\beta$-naphthol undergoes these substitutions in the I position, and so behaves as though it had the other structure (formula 2).
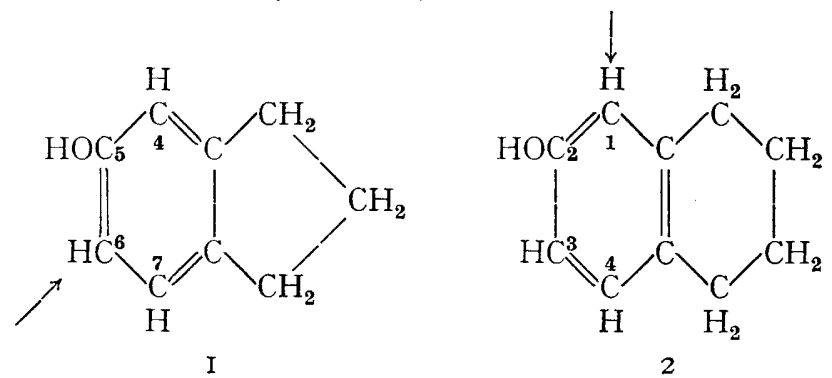

These facts they explained by pointing out that since the angle between two single bonds is less than that between a single bond and a double bond, when the bonds come from a four-covalent van't Hoff-le Bel atom (the angles being $109^{\circ} 28^{\prime}$ and $125^{\circ} 16^{\prime}$, respectively) the angles between successive pairs of external valencies of a single benzene structure should alternate, as shown in Fig. I, angle $\alpha$ being less than angle $\beta$. They then stated that, while in ordinary benzene there could be a tautomerism between the two Kekulé structures with equal concentrations of both present, if two adjacent valencies are pulled together (as they would be if a five-membered ring were attached to them)

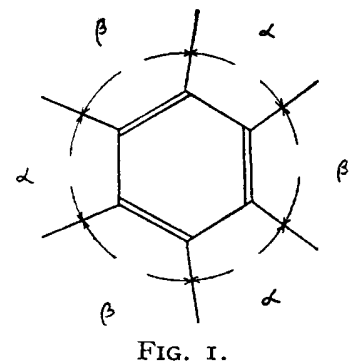
one structure - the one with the single bond between the valencies in question-is made much more stable than the other, and predominates.

1 Mills and Nixon, J. Chem. Soc,, 1930, 2510. 
In this way Mills and Nixon explained the behaviour of 5-hydroxyhydrindene. The reason for the behaviour of $a r$-tetrahydro- $\beta$-naphthol could not be expressed so simply, but they advanced arguments from a comparison of the strains in the two structures to show that when a six-membered ring is attached the structure with the double bond common to the two rings is the more stable, although according to their calculations the difference is small and vanishes if the angle between the valencies of a methylene group is $112.8^{\circ}$.

The conception of the benzene molecule which has been developed from the ideas of wave mechanics calls for some modification of the above interpretation of the facts. Two approximate methods of treatment have been applied to the problem of the structure of benzene, the method of molecular orbitals and the method of valency-bond wave functions ${ }^{2}$; the results of these are essentially in agreement. ${ }^{3}$ In our discussion of the present problem we shall make use of the second method, inasmuch as the first one does not offer an approach to it. It appears very definitely that benzene is a single molecular species and from the second method we can say that it is a hybrid of the two Kekule structures and various excited structures, and that the angles between the pairs of external valencies are all $60^{\circ}$. In the wave function for the whole molecule, the wave functions corresponding to the two Kekule structures occur with equal coefficients, because these structures, regarded as separate entities, have equal energies. If, however, two ortho valencies are displaced from a mutual inclination of $60^{\circ}$, the two Kekule structures no longer correspond to equal energies ; if the angle is increased the structure with the double bond between the ortho valencies in question, hereinafter called structure I, will have the lower energy and its coeffcient will therefore increase, while that of the other structure, hereinafter called structure II, will decrease. The reverse will occur if the angle is decreased. Owing to the great energy corresponding to resonance between the two structures, these changes of coefficient will, however, be relatively small.

In order to treat the problem quantitatively we shall ignore the excited structures (whose contribution to the resonance energy is small), and shall set up and solve the quadratic secular equation for the wave functions of the two Kekule structures. In the simple treatment of benzene, ${ }^{2}$ the matrix elements $\mathrm{H}_{\mathrm{I}, \mathrm{I}}$ and $\mathrm{H}_{\mathrm{II} \text {, II }}$ are both equal to $Q-W+\frac{3}{2} \alpha$, and the elements $\mathrm{H}_{\mathrm{I}}, \mathrm{II}$ and $\mathrm{H}_{\mathrm{II}, \mathrm{I}}$ are equal to $\frac{1}{4}(Q-W)+\frac{3}{2} \alpha, Q$ being the Coulomb energy, $W$ the total energy, and $\alpha$ the exchange integral, which has the value $34,000 \mathrm{cal} / \mathrm{gm}$. mole. This $Q$ includes the strain energy caused by altering the angles of the two ortho valencies from $\phi_{\mathrm{I}}$ or $\phi_{\mathrm{II}}$ to $60^{\circ}$. In the present problem its value is different in the different matrix elements, and therefore it is necessary to express the strain energy explicitly in each. The strain energy in $H_{I, I}$ is that which there would be if the molecule had structure (I) only, i.e., $\frac{1}{2} k\left(\phi-\phi_{1}\right),{ }^{2}$ where $k$ is a force constant and $\phi_{\mathrm{I}}$ is the angle between the unstrained valencies in structure $(I)$. Similarly in $H_{I I}$, II it is $\frac{1}{2} k\left(\phi-\phi_{\mathrm{II}}\right) \cdot^{2}$ In the resonance integrals $\mathrm{H}_{\mathrm{I}, \mathrm{II}}$ and $\mathrm{H}_{\mathrm{II}}$, I it may reasonably be assumed that it is approximately the mean of those in the two structures, i.e.,

$$
\frac{1}{4} K\left[\left(\phi-\phi_{\mathrm{I}}\right)^{2}+\left(\phi-\phi_{\mathrm{II}}\right)^{2}\right] \text {. }
$$

2 Slater, Physic. Rev., r931, 38, г ro9 ; ibid., 37, 489 ; Hückel, Z. Physik., I931, 70, 204; Pauling, J. Chem. Physics, I933, I, 280; Pauling and Wheland, bid., 1933, 1, 362 .

3 Wheland, $J$. Chem. Physics, 1934, 2, 476. 
The secular equation is therefore :

$$
\left|\begin{array}{c}
Q^{\prime}-W+\frac{1}{2} k\left(\phi-\phi_{\mathrm{I}}\right)^{2}+\frac{3}{2} \alpha \\
\frac{1}{4}\left\{Q^{\prime}+\frac{1}{4} k\left[\left(\phi-\phi_{\mathrm{I}}\right)^{2}+\left(\phi-\phi_{\mathrm{II}}\right)^{2}\right]-W\right\}+\frac{3}{2} \alpha \\
\frac{1}{4}\left\{Q^{\prime}+\frac{1}{4} k\left[\left(\phi-\phi_{\mathrm{I}}\right)^{2}+\left(\phi-\phi_{\mathrm{II}}\right)^{2}\right] \frac{-{ }^{2}}{Q^{\prime}-W}+\frac{3}{2} \alpha\right. \\
Q^{\prime}-\frac{1}{2} k\left(\phi-\phi_{\mathrm{II}}\right)^{2}+\frac{3}{2} \alpha
\end{array}\right|=0 .
$$

For numerical roots to be obtained, values must be ascribed to $\phi_{\mathrm{I}}, \phi_{\mathrm{II}}$, and $k$.

The natural values of the angles $\gamma$ (or $\delta^{\prime}$ ) and $\delta$ (or $\gamma^{\prime}$ ) shown in Figs. 2 and 3 , are $109^{\circ} 28^{\prime}$ and $125^{\circ} 16^{\prime}$, respectively. These values cannot be achieved simultaneously because the benzene angle is

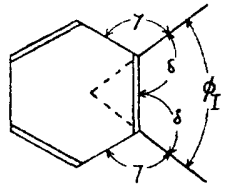

FIg. 2.

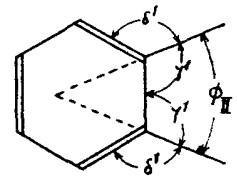

FIG. 3 .

$120^{\circ}$. If we assume that the strain is equally divided between $\gamma$ and $\delta$ and between $\gamma^{\prime}$ and $\delta^{\prime}$, we are led to the values $\phi_{\mathrm{I}}=75.5^{\circ}$ and $\phi_{\mathrm{II}}=44.5^{\circ}$. $k$ is a bending force constant ; since the bending of each bond is one half of the total, say $\frac{\phi-\phi_{I}}{2}$, the total bending energy is $a \frac{\left(\phi-\phi_{I}\right)^{2}}{4}$, where $a$ is the energy necessary to bend one bond through $\mathrm{I}^{\circ}$, and therefore $k=a / 2$. According to Stuart $4 a$ is 16 cal. $/ \mathrm{gm}$. mole. for the $\mathrm{C}-\mathrm{H}$ bond in hydrogen cyanide, and hence $k=8 \mathrm{cals}$./gm. mole. We shall use this value for our $\mathrm{C}-\mathrm{C}$ bonds in default of a better one.

The secular equation has been solved for $Q^{\prime}-W$, with $\phi$ assuming values between $45^{\circ}$ and $75^{\circ}$, and the ratios of the coefficients $\kappa_{\text {I }}$ and $\kappa_{\text {II }}$ of the wave functions for structures $I$ and II in the wave function of the actual molecule, $\psi=\kappa_{\mathrm{I}} \psi_{\mathrm{I}}+\kappa_{\mathrm{II}} \psi_{\mathrm{II}}$, have been evaluated. The resonance energy, $E$, is the difference between $W$ and $Q$ ( $Q$ includes the strain energy), and since it can easily be shown that in this case

$$
W \cong Q+\frac{1}{5} 2 \alpha\left(\mathrm{I}-\frac{3}{20} \delta^{2}\right), \quad \text { where } \delta=\frac{\kappa_{\mathrm{II}}}{\kappa_{\mathrm{I}}}-\mathrm{I},
$$

$E$ can be calculated, and also $\Delta E$, the change of resonance energy relative to that in normal benzene, wherein $\phi=60^{\circ}$ and $\kappa_{\mathrm{II}} / \kappa_{\mathrm{I}}$ is unity. From

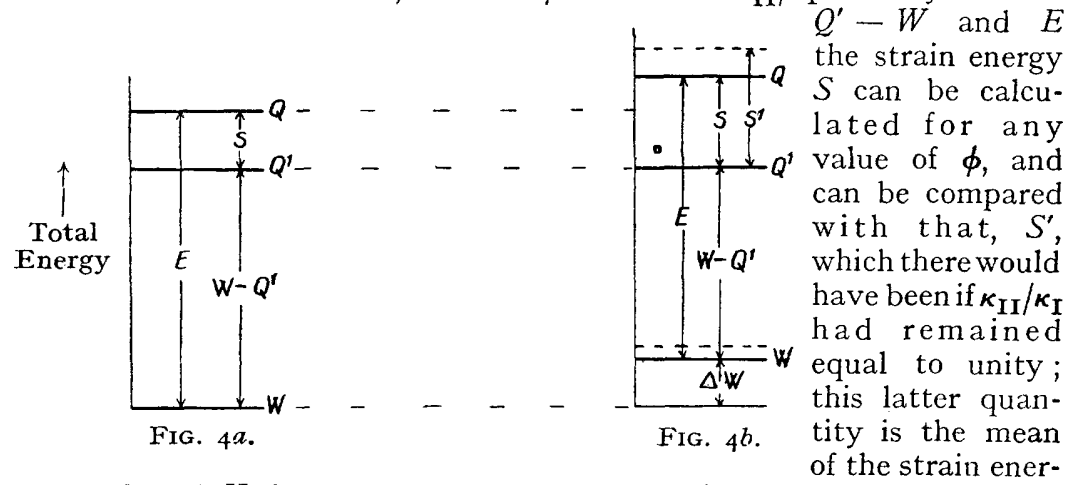

gies in I and II for any particular value of $\phi$, which is equal to the sum of the strain in normal benzene (96I cals./gm. mole.) and the quantity $\frac{1}{2} k\left(\phi-60^{\circ}\right)^{2}$. The stability of the molecule as a whole, relative

${ }^{4}$ Stuart, Molekülstruktur, Springer, Berlin, 1934, p. 86. 
to normal benzene, is given by the differences in total energy, $\Delta W$. The energy relations involved are illustrated in Figs. $4 a$ and $4 b, 4 a$ showing the situation in normal benzene and $4 b$ that in a strained benzene. Fig. $4 a$ is explained by the foregoing and so is most of Fig. $4 b$; the upper broken line, in the latter, shows the value which $Q$ would have if $\kappa_{\text {II }} / \kappa_{I}$ remained equal to unity, and the lower broken line shows what the total energy would have been if also the resonance energy had been the same as in normal benzene. The values of these quantities for a series of values of $\phi$ are collected together in Table I.; the energies are expressed in cals. $/ \mathrm{gm}$. mole.

TABLE I.

\begin{tabular}{|c|c|c|c|c|c|c|c|c|c|}
\hline$\phi$. & $w$ & $\Delta W$. & $\kappa_{I I} / \kappa_{I^{*}}$ & $E$. & $\Delta E$ & $s$. & $s^{\prime}$. & $\Delta S^{\prime}$ & $S^{\prime}-s$. \\
\hline $45^{\circ}$ & $Q^{\prime}-79,784$ & 855 & $x \cdot 063$ & $-8 \mathrm{I}, 552$ & $4^{8}$ & $\mathrm{I}, 768$ & $\mathrm{I}, 86 \mathrm{I}$ & 900 & - \\
\hline $5^{\circ}$ & $\tilde{Q}^{\prime}-80,259$ & 380 & $\mathrm{I} \cdot \mathrm{O}_{4} \mathrm{I}$ & $-81,579$ & 21 & 1,320 & $I, 36 x$ & 400 & $-4 \mathrm{I}$ \\
\hline $55^{\circ}$ & $Q^{\prime}-80,543$ & 96 & 1.020 & $-8 I, 595$ & 5 & 1,052 & $\mathrm{I}, 06 \mathrm{I}$ & 100 & -9 \\
\hline $60^{\circ}$ & $\tilde{Q}^{\prime}-80,639$ & o & $1 \cdot 000$ & $-81,600$ & o & $96 \mathrm{I}$ & $96 \mathrm{I}$ & o & \\
\hline $65^{\circ}$ & $\tilde{Q}^{\prime}-80,543$ & 96 & 0.980 & $-8 \mathrm{I}, 595$ & 5 & 1,052 & $\mathrm{I}, 06 \mathrm{I}$ & 100 & -9 \\
\hline $70^{\circ}$ & $\tilde{Q}^{\prime}-80,259$ & 380 & 0.960 & $-8 \mathbf{r}, 579$ & 21 & 1,320 & $\mathrm{I}, 36 \mathrm{r}$ & 400 & $-4 I$ \\
\hline $75^{\circ}$ & $\widetilde{Q}^{\prime}-79,784$ & 855 & $0.94^{\mathrm{I}}$ & $-81,55^{2}$ & $4^{8}$ & $x, 768$ & $I, 86 \mathrm{I}$ & 900 & -93 \\
\hline
\end{tabular}

It will be observed that the resonance energy is decreased numerically by the change in $\kappa_{\mathrm{II}} / \kappa_{\mathrm{I}}$, but that the strain energy is decreased about twice as much by this same cause, so that the nett result is that the destabilisation of the molecule by the bending of the bonds is slightly less than it would have been if $\kappa_{I I} / \kappa_{I}$ had remained equal to unity or if there had been no resonance at all.

The changes in $\kappa_{\mathrm{II}} / \kappa_{\mathrm{I}}$ are relatively small, and it remains to discuss whether or not they could account for the experimental facts. If a fivemembered ring is attached to two ortho valencies of benzene the sum of the bond angles has to decrease from $568^{\circ} 24^{\prime}$ to $540^{\circ}$, and if the simplifying assumption be made that the reduction of each bond angle is equal, this means that $\phi$ is reduced by about $12^{\circ}$, i.e., to about $48^{\circ}$, and therefore that $\kappa_{\mathrm{II}} / \kappa_{\mathrm{I}}$ is approximately $\mathrm{I} \cdot 05$. This 5 per cent. change in the ratio of the coefficients of the two Kekule structures is small. It may well be large enough, however, to cause one of the two substitution reactions to be favoured over the other to the extent shown by experiment. Thus we need only recall the important effects of substituents in the benzene ring in influencing the rates of further substitution in order to obtain support for the view that a small influence exerted on the benzene ring can produce important changes in rates of reaction. It is not unreasonable to expect that the effect consists primarily in a change in the heat of activation. If we represent the activation energy for the reaction of substitution in position 4 of the hydrindene derivatives by $A_{\mathrm{I}}$ and that for position 6 by $A_{\text {II }}$ (these corresponding to positions I and 3 of the tetrahydronaphthalene derivatives), we may tentatively assume, in order to make a quantitative calculation, that the ratio $A_{\mathrm{I}} / A_{\mathrm{II}}$ is given by the equation

$$
\frac{A_{\mathrm{I}}}{A_{\mathrm{II}}}=\left(\frac{\kappa_{\mathrm{II}}}{\kappa_{\mathrm{I}}}\right)^{2}
$$

the occurrence of the second power being characteristic of wavemechanical calculations. In the absence of pronounced steric effects, it 
is probable that the ratio of the rates $r_{I}$ and $r_{I I}$ of the reactions is given by the expression

$$
\frac{r_{\mathrm{II}}}{r_{\mathrm{I}}}=\frac{e^{-A_{\mathrm{II}} / R T}}{e^{-A_{\mathrm{I}} / R T}}, \quad \text { whence } \frac{r_{\mathrm{II}}}{r_{\mathrm{I}}}=e^{A_{\mathrm{II}}\left[\left(\frac{\kappa_{\mathrm{II}}}{\kappa_{\mathrm{I}}}\right)^{2}-1\right] / R T}
$$

Since a 5 per cent. increase in $\kappa_{\mathrm{II}} / \boldsymbol{\kappa}_{\mathrm{I}}$ produces a Io per cent. increase in the square, it follows that the ratio anticipated for the rates of reaction would be $e^{\mathbf{0 \cdot 1} \boldsymbol{A}_{\mathrm{II}} / \boldsymbol{R T}}$, and if $A_{\mathrm{II}}$ lies between 10,000 and 20,000 cals. $/ \mathrm{gm}$. mole., as is probable, the rate ratio is between 5 and 27 , approximately. Thus, since the ratio of the quantities of product is that of the rates of reaction, the product of reaction II would form between 83 per cent. and $96 \cdot 5$ per cent. of the total yield.

The prediction of the effect of attaching a six-membered reduced ring is less simple, owing to the more complex steric relations. Our procedure here is different from that of Mills and Nixon, ${ }^{1}$ for they assumed that the molecule had either structure I or structure II, whereas we consider that initially it has the symmetrical resonating structure and then calculate how much it is distorted when a ring is attached. The reduced ring cannot have a stable configuration in which it is coplanar with the benzene ring: the carbon atoms 6 and 7 may either be both on one side of this plane or one on either side. Assuming the carbon-carbon distances $^{5}$ to be $\mathrm{I} \cdot 39 \AA$ in the benzene ring and I.54 $\AA$ elsewhere, it is found on calculation that the ortho valency angle is decreased for the first configuration and increased for the second. The resultant total strain energy is less for the second than for the first configuration by about $300-500$ cal./gm. mole. Moreover, it is known empirically that cyclohexane derivatives ${ }^{6}$ and $I \cdot 4$-dioxane ${ }^{7}$ exist predominantly in the " $Z$ " or chair form, presumably because of steric repulsion of the hydrogen atoms of the methylene groups. Therefore it is very probable that tetrahydronaphthalene and its derivatives also exist predominantly in the second configuration, which is approximately a " $Z$ " configuration. For this configuration the angle $\phi$ is increased by $10^{\circ}$ to $70^{\circ}, \kappa_{\text {II }} / \kappa_{I}$ becomes 0.96 , and the ratio of the rate of reaction I to that of reaction II will, on the previous hypothesis, be $e^{0.08 A_{\mathrm{I}} / k T}$, which for values of $A_{\mathrm{I}}$ between 10,000 and 20,000 cals./gm. mole. is 3.8 to 14.5 , so that the product from reaction I should form from 79 per cent. to 93.5 per cent. of the total yield.

The exact experimental facts as adduced by Mills and Nixon are the following. The bromination of 5-hydroxyhydrindene goes smoothly to give the 6-bromo derivative, with very little, if any, by-product, and 90 per cent. of the coupling of it with diazotised $p$-chloroaniline takes place in the 6 position; the coupling with diazotised $p$-toluidine also occurs mainly in the 6 position but gives a small quantity of by-product which although not identified completely is isomeric with the main product. $^{1}$ Bromination of 5 -acetoamido-hydrindene yields the 6 -bromo derivative only, judging by the description of the reaction given by Borsche and Bodenstein. ${ }^{8}$ The only product isolated in the bromination

5 Pauling and Brockway, J. Chem. Physics, 1934, 2.

6 Dickinson and Bilicke, J. Am. Chem. Soc., I928, 50, 764; Hassel, Trans. Favaday Soc., I934, 30, 874 .

${ }^{7}$ Schwingel and Greene, J. Am. Chem. Soc., 1934, 56, 653; Sutton and Brockway, ibid., 1935, 57, 473.

8 Borsche and Bodenstein, Ber., 1926, 59, I910. 
of $a r$-tetrahydro- $\beta$-naphthol is the $\mathrm{I}$-bromo derivative the structure of which was decided by elimination rather than by direct determination. ${ }^{9}$ Coupling with diazotised sulphanilic acid occurs only in the I position; no definite mono-nitro derivative can be prepared because of the ease with which poly-nitration occurs; sulphonation is anomalous, occurring readily and completely in the 3 position. ${ }^{9}$ Two mono-bromo derivatives are obtained directly from 2 -acetamido-tetrahydronaphthalene, one in 82 per cent. yield and the other in 12 per cent.; ${ }^{10}$ one must have the bromine atom attached to position $I$ and the other must have it on position 4, but there is no direct chemical evidence to show which is which. It should be observed, however, that since the base derived from the main product is more volatile in steam than the other, it must certainly have the bromine atom on the I position, i.e., ortho to the amino group, because $o$-chloro and $o$-bromoanilines are more volatile than the $m$ - or $p$-compounds. ${ }^{11}$ Thus it may be said that the brominations and diazo couplings go to the extent of 90 per cent. or more in the 4 position of the hydrindene derivatives, and to a similar extent in the I position of the tetrahydronaphthalene derivatives. There is, therefore, good agreement between the experimental facts and the theoretical predictions which have just been made. This shows that the wavemechanical concept of the structure of the benzene molecule is capable of accounting for the facts interpreted by Mills and Nixon in terms of stabilisation of the individual Kekule structures. It may be pointed out also that our treatment is based on the old contention that for some purposes, and within limitations, the substitution reactions of benzene derivatives can be discussed by considering the properties of the double bonds of the Kekule structures.

One further question which merits discussion is that of the effect which forcibly altering the an gle between two ortho valencies would have upon the angles between the other four. On the classical i deas it would obviously be expected that if the angle between one pair were fixed at $\phi$ then the angles between the alternate pairs would also be $\phi$. Using wave-mechanical ideas the method of answering the question is the following. The expression for the total energy $W$,

$$
W=\frac{\int\left(\kappa_{\mathrm{I}} \psi_{\mathrm{I}}+\kappa_{\mathrm{II}} \psi_{\mathrm{II}}\right) H\left(\kappa_{\mathrm{I}} \psi_{\mathrm{I}}+\kappa_{\mathrm{II}} \psi_{\mathrm{II}}\right) d \tau}{\int\left(\kappa_{\mathrm{I}} \psi_{\mathrm{I}}+\kappa_{\mathrm{II}} \psi_{\mathrm{II}}\right)^{2} d}
$$

consists, when evaluated, of the Coulomb terms and exchange terms. The sum of the former terms has already been subdivided into two parts, the strain energy of a pair of external valencies, and the remainder of the energy, but this latter can be further subdivided, inasmuch as it also c on tains strain energy terms for the other two pairs of external valencies, the sum of which is

$$
\frac{2\left\{\kappa_{\mathrm{I}}^{2} \frac{k}{2}\left(\phi^{\prime}-\phi_{\mathrm{I}}\right)^{2}+\frac{\kappa_{\mathrm{I}} \kappa_{\mathrm{II}}}{2} \frac{k}{4}\left[\left(\phi^{\prime}-\phi_{\mathrm{I}}\right)^{2}+\left(\phi-\phi_{\mathrm{II}}\right)^{2}\right]+\kappa_{\mathrm{II}}^{2}-{ }_{2}\left(\phi^{\prime}-\phi_{\mathrm{II}}\right)^{2}\right\}}{\kappa_{\mathrm{I}}^{2}+\frac{1}{2} \kappa_{\mathrm{I}} \kappa_{\mathrm{II}}+\kappa_{\mathrm{II}}^{2}},
$$

$\phi^{\prime}$ being the angle between either of the two alternate pairs. In order to get the minimum energy, when $\kappa_{\mathrm{II}} / \kappa_{\mathrm{I}}$ has been fixed by the forcible

- Schroeter, Annalen, 1922, 426, 83 .

10 Smith, $J$. Chem. Soc., 1904, 85, 730.

11 Sidgwick and Rubie, J. Chem. Soc., 1921, 119, ror 3 ; Fittig and Mager, Ber., I874, 7, I179; ibid., I875, 8, 364; Nemurowski, Ber., r891, 24, ref. 271 ; Holleman, Rec. Trav. chim. Pavs Bas, I906, 25, I 86 . We are indebted to Professo H. J. Lucas for suggesting this line of argument. 
bending of one pair, this sum must be minimised with respect to $\phi^{\prime}$, and from the differential relation which we thereby obtain the equilibrium value of $\phi^{\prime}$ for any value of $\kappa_{\text {II }} / \kappa_{\text {I }}$ can be calculated :-

$$
\phi^{\prime}=\frac{1}{2}\left(\phi_{\mathrm{I}}+\phi_{\mathrm{II}}\right)+\frac{\mathrm{I}-\left(\kappa_{\mathrm{II}} / \kappa_{\mathrm{I}}\right)^{2}}{2+\left(\kappa_{\mathrm{II}} / \kappa_{\mathrm{I}}\right)+2\left(\kappa_{\mathrm{II}} / \kappa_{\mathrm{I}}\right)^{2}}\left(\phi_{\mathrm{I}}-\phi_{\mathrm{II}}\right) .
$$

For $\phi_{\mathrm{I}}=75.5^{\circ}, \phi_{\mathrm{II}}=44.5^{\circ}$, and $\kappa_{\mathrm{II}} / \kappa_{\mathrm{I}}=\mathrm{I} \cdot 063, \phi^{\prime}$ is found to be $59^{\circ} \mathrm{I} 5^{\prime}$, and for $\kappa_{\mathrm{II}} / \kappa_{\mathrm{I}}=0.94 \mathrm{I}, \phi^{\prime}$ is $60^{\circ} 45^{\prime}$. It is seen that the bending of two valencies affects the disposition of the other four to only a very small extent. There are at present no published data by which this conclusion can be tested, and since the introduction of substituents in order to obtain such data from, say, measurements of electric dipole moment or electron-diffraction photographs would usually cause complications, as most substituents set up resonance of types other than that considered above, the interpretation of the results might be by no means straightforward.*

\section{Summary.}

The Mills-Nixon effect has been examined by a very simple wavemechanical treatment. This has led to the conclusion that the effect of saturated side rings upon the ratio of the coefficients of the wave-functions of the two Kekule structures is relatively small, being not more than about 6 per cent., and that the benzene ring retains the greater part of its stabilising resonance energy. Nevertheless, making the reasonable assumption that the ratio of the activation energies, for reaction as either one of the two Kekule structures, depends upon the square of the ratio of coefficients, it is possible to account for the experimental facts. The effect which bending two valencies has upon the angles between the other valencies projecting from the benzene ring is found to be very small.

\section{The Gates Chemical Laboratory, California Institute of Technology, Pasadena, California.}

* It may be pointed out, however, that although certain complications might be expected to arise in the previous considerations relating to the phenolic derivatives, there was no apparent indication of this happening. 